\title{
Improved Meshfree Approach to The Solution of Sixth Order Differential Equations
}

\author{
Muhammad Azam*, Khalid Parvez and Muhammad Omair \\ Research Center for Modeling and Simulation, National University of Sciences and Technology, Islamabad, Pakistan
}

Received: 28 Jul. 2013, Revised: 30 Oct. 2013, Accepted: 31 Oct. 2013

Published online: 1 Jul. 2014

\begin{abstract}
Numerical Mesh-free method with improved numerical integration using block pulse function (BPF)/Chebyshev wavelets $(\mathrm{CW})$, is engaged for the solution of sixth order boundary value problems (BVP). Moving Least Squares (MLS) approach is used to construct shape functions with optimized weight functions and basis. The proposed improved Element Free Galerkin (EFG) technique has already been successfully implemented on various physical applications in fluids and structures such as solution for large deformations, stresses, strains involving friction, viscosity and viscoelasticity. Numerical results for test cases of sixth order boundary value problems are presented in this article to elaborate the relevant features and of the proposed technique. Comparison with existing techniques shows that our proposed method provides better approximation at reduced computational cost.
\end{abstract}

Keywords: Meshfree; Block Pulse Function; Chebyshev wavelets; Moving Least Squares; Element Free Galerkin; Sixth order boundary value problem

\section{Introduction}

The numerical solutions of sixth order boundary value problems rarely exist in the literature. Such problems are found in astrophysics[1,2,3,4], e.g. the narrow convecting layers bounded by stable layers, which are believed to be surrounding the A-type stars, is modeled by sixth-order differential equation. Such equations handle dynamo action in some stars. Chandrasekhar [5] determined that instabilities are found when an infinite horizontal layer of fluid is heated from below and is under the action of rotation. When these instabilities are treated as ordinary convection, they are modeled by a sixth order ordinary differential equation. Twizell developed a second-order method for solving special and general sixth-order problems [6] and in later work Twizell and Boutayeb developed finite-difference methods of order two, four, six and eight for solving such problems [7]. Siddiqi and Twizell used sixth-degree splines $[8,9,10]$, where spline values at the mid knots of the interpolation interval and the corresponding values of the even order derivatives are related through consistency relations. M. E Gamel et al. used Sinc-Galerkin method for the solutions of sixth order boundary-value problems [11]. Siraj-ul-islam et al solved Sixth-Order Boundary-Value
Problems using Non-Polynomial Splines Approach [12] and Wazwaz $[13,14]$ used decomposition and modified domain decomposition methods to explore solution of the sixth-order boundary-value problems. In this study, Element Free Galerkin (EFG) technique with Block Pulse Function (BPF) and Chebyshev wavelets (CW) based numerical integration is applied to obtain smooth approximations for the following boundary-value problem $[11,13,15,17]$.

$$
\frac{d^{6} u}{d x^{6}}+k(x) u(x)=f(x), a<x<b
$$

With boundary condition, $u^{i}(0)=u^{i}$

Where $\mathrm{u}(\mathrm{x})$ and $\mathrm{f}(\mathrm{x})$ are continuous functions defined in the interval $[\mathrm{a}, \mathrm{b}]$. It is considered that $f(x) \in C^{6}[a, b]$ is real.

The development of numerical methods for the solution of these differential equations has attracted the attention of researchers for the last many decades. Galerkin-Finite element method (FEM) is one of the most popular and well developed numerical methods. FEM has been extensively used in the computational mechanics, due to its robustness, versatility and convenience. However, FEM has inherent problems such as locking and poor derivative solutions [18]. Finite element method

\footnotetext{
${ }^{*}$ Corresponding author e-mail: azam.rcms.nust@gmail.com
} 
requires considerable amount of work and time in generation of a predefined mesh of the problem domain. In structural analysis, the stresses calculated employing Finite element method algorithms are discontinuous and less accurate. When large deformations are analyzed, Distortions in elements cause loss in solution accuracy in the case of large deformations $[19,20]$. Other numerical techniques such as, the boundary element method (BEM) have emerged over the period of time but the BEM is limited by the availability of the infinite space fundamental solution for (at least the highest linear) differential operator of the problem. Atluri, S.N., Zhu and G.R.Liu [18,21] used Meshfree method instead of FEM for large deformations in physical problems. A meshfree (or meshless) method is an alternate method where system algebraic equations for the whole problem domain are established without the use of a predefined mesh for the domain discretization [21]. Meshfree method presents an attractive option for the solution of such problems due to its flexibility in absence of nodal interconnectivity. Several Meshless techniques have been developed, e.g. the Diffuse element method (Nayroles et al., 1992), the Element Free Galerkin (EFG) method (Belytschko et al., 1994b), Meshless local Petrov-Galerkin (MLPG) method, (Zhu and Atluri, 1998), the Smooth Particle Hydrodynamics (SPH) method, the Reproducing Kernel Particle method (Liu et al., 1993), Boundary Node method (Mukherjee and Mukherjee, 1997a,b) and Boundary Point Interpolation methods (Liu et al., 2000d; $\mathrm{Gu}$ and Liu, G.R., 2001a,e) [18, 19,20,21]. These approximate solution techniques have helped mathematicians, physicists and engineers to analyze complex phenomena in fluid mechanics at reduced computational costs. In this study, we propose a novel numerical technique for the solution of sixth order boundary value problems using improved Element Free Galerkin method with Block Pulse Function (BPF) and Chebyshev wavelets $(\mathrm{CW})$ based numerical integration.

\section{Numerical Technique}

Meshless techniques maintain the local character of the numerical implementation, by using a local interpolation to represent the trial function with the fictitious values of the unknown variable at some randomly located nodes. The local interpolation techniques generally used are moving least square method, partition of unity method, reproducing kernel method, hp-clouds, Shepard function etc. The moving least squares approximation has reasonably high accuracy and can be generalized to work with n-dimensional problems. It was developed for interpolation, data fitting and surface construction. It provides continuous approximation for the field function over the entire problem domain $[18,21]$. The numerical technique used in this study employed Moving Least Square (MLS) method to generate the shape functions with Gaussian weight function. Shape functions for meshless techniques need to satisfy certain conditions such as adherence to partition of unity $\left[\sum_{i=1}^{n} \Phi_{I}(x)=1\right]$, compact domain of influence, adapt to randomness of nodes, to name a few. Computational efficiency is significantly affected by the choice of shape function. Then Galerkin weak form of the boundary value problem was formulated to give the system equations. The boundary conditions were successfully imposed using Penalty/Lagrange method. Both Block-Pulse Function and Chebyshev wavelets were used to perform numerical integration of the system equation.

MLS approximation $u^{h}(x)$ of a field variable $u(x)$, is defined as:

$$
u^{h}(x)=\sum_{j=1}^{m} p_{j}(x) a_{j}(x)=p^{T}(x) a(x), \forall x \varepsilon \Omega
$$

Where,

$$
\begin{gathered}
\mathrm{m}=\text { number of terms in the basis, } \\
p_{j}(x)=\text { polynomial basis function, } \\
a_{j}(x)=\text { non-constant coefficients, } \\
p^{T}(x)=\left[p_{1}(x), p_{2}(x), p_{3}(x), \cdots p_{m}(x)\right]
\end{gathered}
$$

$p^{T}(x)$ is a complete monomial basis vector of order $\mathrm{m}$; $\mathrm{a}(\mathrm{x})$ is a vector containing coefficients: $a_{j}(x), j=1,2,3 \cdots m$. local approximation at a point $x_{I}$. The unknown coefficients of approximation are computed by minimizing the difference between the and the nodal parameter $u_{I}$ for the node $I$, i.e. $u_{I}=u\left(x_{I}\right)$

Here the sample point $x_{I}$ may be a nodal point under consideration or a quadrature point. The support of the nodal point $x_{I}$ is usually taken to be a circle of radius $r_{i}$, centered at $x_{I}$. Weighted residual functional is:

$$
J=\sum_{i=1}^{n} w\left(x-x_{I}\right)\left(u^{h}(x)-u_{I}\right)^{2}
$$

Where is weight function $w\left(x-x_{I}\right)$ associated with the node $I$ calculated at point $x . w\left(x-x_{I}\right)>0$ for all points $x$ in the support domain of node $I$, and

$$
u^{h}\left(x_{l}\right)=p^{T}\left(x_{l}\right) a(x)
$$

Minimization of weighted residual functional results in:

$$
\begin{gathered}
A(x) a(x)=B(x) u \\
a(x)=A^{-1}(x) B(x) u \\
u=\left[u_{1}, u_{2}, u_{3}, \cdots u_{n}\right]
\end{gathered}
$$

The weight moment matrix $A(x)$ is:

$$
A(x)=p^{T} w p=\sum_{i=1}^{n} w\left(x-x_{I}\right) p\left(x_{I}\right) p^{T}\left(x_{I}\right)
$$


$B(x)$ is defined as:

$$
B(x)=p^{T} w
$$

where $P$ is:

$$
P=\left[p^{T}\left(x_{1}\right), p^{T}\left(x_{2}\right), p^{T}\left(x_{3}\right), \cdots, p^{T}\left(x_{n}\right)\right]^{\prime}
$$

$\mathrm{W}$ is the weight function matrix:

$$
W=\left[\begin{array}{ccc}
w\left(x-x_{1}\right) & \cdots & 0 \\
\vdots & \ddots & \vdots \\
0 & \cdots & w\left(x-x_{n}\right)
\end{array}\right]
$$

Following Gaussian weight function is used in the present study:

$$
\begin{gathered}
w\left(x-x_{I}\right)=-16 / \alpha^{16}\left(16\left(x-x_{I}\right)^{8}\right. \\
-224\left(x-x_{I}\right)^{6} \alpha^{2}+840\left(x-x_{I}\right)^{4} \alpha^{2} \\
\left.+105 \alpha^{8}\right) \operatorname{Exp}\left(-1 / \alpha^{2}\left(x-x_{I}\right)^{2}\right)
\end{gathered}
$$

Shape perimeter $\alpha_{c}$ substantially affects the accuracy of the solution. Figures 1 and 2 illustrate weight function for different values of shape parameters and its derivatives.

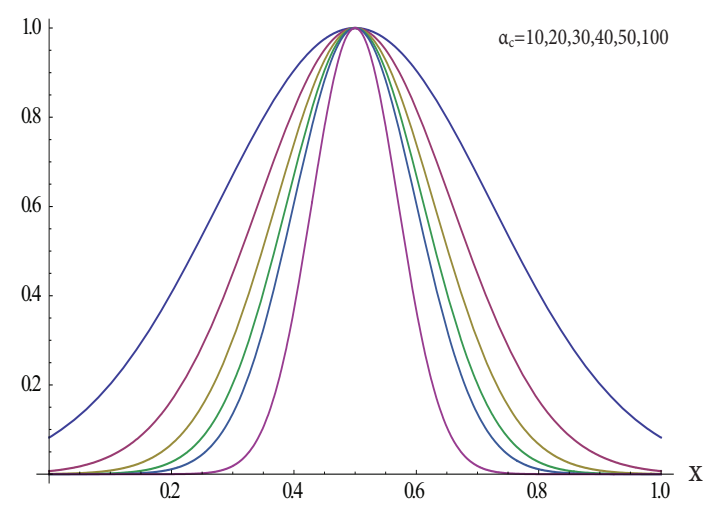

Fig. 1: Weight functions in the support domain

Solving equation (6) for $a(x)$ and substituting in equation (4)results in:

$$
u^{h}(x)=\Phi^{T}(x) \cdot \mathbf{u}=\sum_{I=1}^{n} \phi\left(x_{I}\right) u_{I} \quad \forall x \in \Omega
$$

$\Phi(x)$ is the nodal shape function.

$$
\Phi(x)=\left[\phi_{1}(x), \phi_{2}(x), \cdots, \phi_{n}(x)\right]
$$

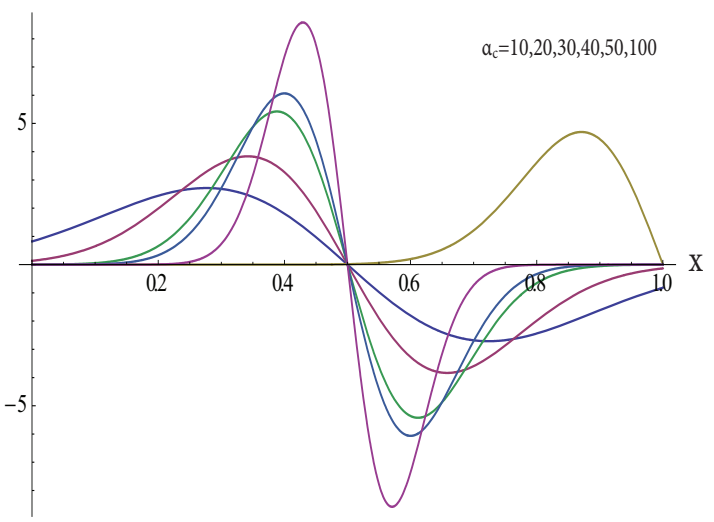

Fig. 2: Derivative of weight functions in the support domain

For any node I,

$$
\Phi_{I}(x)=p^{T}(x)\left(A^{(-1)}(x) B(x)\right)_{I}
$$

Next we transform the strong form of sixth order boundary value problem (1) into symmetric variational (weak) form.

$$
\int_{a}^{b}\left(\frac{d^{6} u}{d x^{6}}+k(x) u(x)-f(x)\right) v d x=0
$$

Here $u$ is the trial function and $v$ is the test function. We get the following linear equation system after employing the 'Integration by parts' technique and substitution of the shape functions and boundary conditions:

$$
\begin{gathered}
\int_{a}^{b}\left(\Phi^{\prime \prime \prime}\left(\Phi^{\prime \prime \prime}\right)^{T}+k(x) \Phi \Phi^{T}\right) d x \hat{\mathbf{u}}=\int_{a}^{b} f \Phi d x \\
K \hat{\mathbf{u}}=F \\
K=\int_{a}^{b}\left(\Phi^{\prime \prime \prime}\left(\Phi^{\prime \prime \prime}\right)^{T}+k(x) \Phi \Phi^{T}\right) d x \\
F=\int_{a}^{b} f \Phi d x
\end{gathered}
$$

Nodal fictitious values $\hat{\mathbf{u}}$ are obtained by solving the above system of equations. Product of nodal fictitious values and shape functions $\Phi$ results in required numerical solution:

$$
\mathbf{u}=\hat{\mathbf{u}} \Phi
$$




\subsection{Element Free Galerkin Technique(EFG)}

Element Free Galerkin technique was introduced by Belytschko et al.[22]. EFG results in generation of nodes with variable interconnectivity, thus providing huge flexibility in discretization. Solution is expanded in a basis of MLS shape functions instead of piecewise polynomial basis as in case of the Finite element method. System equations are formulated using Galerkin weak form whereas integration is performed using background cells. EFG is conforming due to the use of consistent and compatible shape functions $[18,21]$.

\subsection{Numerical Integration}

The accuracy of numerical approximation is dependent on the integration technique. Conventional Gaussian quadrature scheme involves large number of points for accurate numerical solution. In other numerical integration techniques such as Newton-Cotes quadrature rule, fine discretization leads to erroneous results due to high degree of polynomial interpolation [23]. Numerical Integration based on wavelets is becoming popular in recent years [24]. Wavelet transforms were primarily developed for signal analysis, but it has also been used in applications like image compression, data compression, de-noising data and many more. In this study, Block-Pulse and Chebyshev wavelets have been used due to their suitable properties and exceptional performance in numerical integration. Their performance particularly, in approximation of highly oscillatory and improper integral is more accurate as compared to existing method in literature $[25,26]$.

\section{Block-Pulse Function}

Block-Pulse wavelet is one of the simplest wavelet. It is an orthonormal wavelet transform with compact support. Block-Pulse function (BPF) with $\mathrm{m}$ number of sets is defined as [23]:

$$
\phi_{i}(t)= \begin{cases}1 & \text { for } \frac{(i-1) T}{m} \leq t<\frac{i T}{m} \\ 0 & \text { otherwise }\end{cases}
$$

Here $t \in[0, T)$. Also, $i=0,1,2, \ldots, m$ and $h=T / m$. Let us consider the integrand:

$$
\int_{a}^{b} g(x) d x
$$

For $\mathrm{x}=(\mathrm{b}-\mathrm{a}) \mathrm{t}+\mathrm{a}$, we get:

$$
\int_{a}^{b} g(x) d x=(b-a) \int_{0}^{1} g((b-a) t+a) d t
$$

\section{Theorem:}

The integral can be approximated as:

$$
\int_{0}^{1} g(t) d t \approx \frac{1}{m} \sum_{i=1}^{m} g_{i}
$$

Proof:

$$
\int_{0}^{1} g(t) d t \approx \sum_{i=1}^{m} g_{i} \int_{0}^{1} \phi_{i}(t) d t=\frac{1}{m} \sum_{i=1}^{m} g_{i}
$$

To calculate the coefficients $g_{i}$, we consider the nodal points,

$$
t_{k}=\frac{2 k-1}{2 m}, \quad k=1,2, \ldots, m
$$

The equation can be discretized to give:

$$
g\left(t_{k}\right)=\sum_{i=1}^{m} g_{i} \phi_{i}\left(t_{k}\right)=g_{k}, \quad k=1,2, \ldots, m
$$

Therefore, Block-Pulse Function based numerical integration with $\mathrm{m}$ sets, may be evaluated as:

$$
\begin{gathered}
\int_{0}^{1} g(t) d t \approx \frac{1}{m} \sum_{i=1}^{m} g\left(\frac{2 i-1}{m}\right) \\
\int_{a}^{b} g(x) d x \cong \frac{b-a}{m} \sum_{i=1}^{m} g\left(a+(b-a)\left(\frac{2 i-1}{m}\right)\right)
\end{gathered}
$$

\section{Chebyshev Wavelets}

The Chebyshev wavelet of $m$ degree of polynomials of the first kind is defined on interval $[0,1)$ as $[23]$ :

$$
\Gamma_{n, m}(x)= \begin{cases}2^{\frac{k}{2}} \widetilde{\Lambda}_{m}\left(2^{k} x-2 n+1\right) & \frac{n-1}{2^{k}-1} \leq x<\frac{n}{2^{k}-1} \\ 0 & \text { otherwise }\end{cases}
$$

where

$$
\begin{gathered}
m=0,1, \ldots, M-1, \\
n=1,2, \ldots, 2^{k-1},
\end{gathered}
$$

$\mathrm{M}$ and $\mathrm{k}$ are positive integers

and

$$
\widetilde{\Lambda}_{m}(t)= \begin{cases}\frac{1}{\sqrt{\pi}} & m=0, \\ \sqrt{\frac{2}{\pi}} \Lambda_{m}(t) & m>0 .\end{cases}
$$

The coefficients used here are for orthonormality. $\Lambda_{m}(t)$ denotes the Chebyshev polynomials of the first kind of degree $\mathrm{m} . \Lambda_{m}(t)$ and Weight function $W(t)=\frac{1}{\sqrt{1-t^{2}}}$ are 
orthogonal on the interval $[-1,1]$.

Any square integrable function $\mathrm{g}(\mathrm{x})$ in the interval $x \in[0,1)$, can be written as:

$$
\begin{aligned}
& g(x)=\sum_{n=1}^{\infty} \sum_{m=0}^{\infty} c_{n, m} \Gamma_{n, m}(x) \quad x \in[0,1), \\
& g(x) \simeq \sum_{n=1}^{2^{k-1}} \sum_{m=0}^{M-1} c_{n, m} \Gamma_{n, m}(x) \quad x \in[0,1) .
\end{aligned}
$$

Consider the nodal points such that:

$$
x_{p}=\frac{2 p-1}{2^{k} M} \quad p=1,2,3, \ldots, 2^{k-1} M .
$$

Then we have,

$$
g\left(x_{p}\right)=\sum_{n=1}^{2^{k-1}} \sum_{m=0}^{M-1} c_{n, m} \Gamma_{n, m}\left(x_{p}\right) \quad p=1,2,3, \ldots, 2^{k-1} M .
$$

Now, let us consider the integrand, $\int_{a}^{b} g(x) d x$.

For $x=(b-a) t+a$, we get:

$$
\int_{a}^{b} g(x) d x=(b-a) \int_{0}^{1} g((b-a) t+a) d t
$$

\section{Theorem:}

The integral can be approximated as:

$$
\int_{0}^{1} g(x) d x \simeq \frac{2^{1-\frac{k}{2}}}{\sqrt{\pi}} \sum_{n=1}^{2^{k-1}}\left[c_{n, 0}+\sum_{l=1}^{\frac{M-1}{2}} \frac{\sqrt{2}}{1-4 l^{2}} c_{n, 2 l}\right]
$$

Proof.

$$
\begin{aligned}
\int_{0}^{1} g(x) d x & \simeq \sum_{n=1}^{2^{k-1}} \sum_{m=0}^{M-1} c_{n, m} \int_{0}^{1} \Gamma_{n, m}(x) d x, \\
\int_{0}^{1} \Gamma_{n, m}(x) d x & =2^{\frac{k}{2}} \int_{\frac{n-1}{2^{k-1}}}^{2^{k-1}} \widetilde{\Lambda}_{m}\left(2^{k} x-2 n+1\right) d x \\
& =2^{\frac{-k}{2}} \int_{-1}^{1} \widetilde{\Lambda}_{m}(t) d t,
\end{aligned}
$$

since,

$$
\int_{-1}^{1} \Lambda_{m}(t) d t= \begin{cases}0 & \mathrm{~m} \text { is odd } \\ \frac{-2}{m^{2}-1} & \mathrm{~m} \text { is even }\end{cases}
$$

As,

$$
\int_{0}^{1} \Gamma_{n, m}(x) d x= \begin{cases}\frac{2^{1-\frac{k}{2}}}{\sqrt{\pi}} & \mathrm{m}=0, \\ 0 & \mathrm{~m} \text { is odd, } \\ \frac{2^{1-\frac{k}{2}}}{1-m^{2}} \sqrt{\frac{2}{\pi}} & \mathrm{m} \text { is even. }\end{cases}
$$

Therefore,

$$
\int_{0}^{1} g(x) d x \simeq \frac{2^{1-\frac{k}{2}}}{\sqrt{\pi}} \sum_{n=1}^{2^{k-1}}\left[c_{n, 0}+\sum_{l=1}^{\left[\frac{M-1}{2}\right]} \frac{\sqrt{2}}{1-4 l^{2}} c_{n, 2 l}\right]
$$

We know from the definition of Chebyshev wavelets that:

$$
\frac{n-1}{2^{k-1}} \leq \frac{2 p-1}{2^{k} M}<\frac{n}{2^{k-1}},
$$

Therefore, $p=(n-1) M+i$,

$$
i=1,2,3, \ldots, M \text {, }
$$

$$
\begin{gathered}
g\left(x_{p}\right)=g\left(\frac{2(n-1) M+2 i-1}{2^{k} M}\right), \quad i=1,2,3, \ldots, M, \\
\Gamma_{n, 0}\left(x_{p}\right)=\frac{2^{\frac{k}{2}}}{\sqrt{\pi}}, \\
\Gamma_{n, m}\left(x_{p}\right)=\sqrt{\frac{2}{\pi}} 2^{\frac{k}{2}} \Lambda_{m}\left(2^{k} x_{p}-2 n+1\right) \\
=\sqrt{\frac{2}{\pi}} 2^{\frac{k}{2}} \Lambda_{m}\left(\frac{2 i-1}{M}-1\right)
\end{gathered}
$$

So the above system of equations may be written as:

$$
\begin{aligned}
g\left(\frac{2(n-1) M+2 i-1}{2^{k} M}\right)= & \frac{2^{\frac{k}{2}}}{\sqrt{\pi}} c_{n, 0}+\sum_{m=1}^{M-1} \sqrt{\frac{2}{\pi}} 2^{\frac{k}{2}} \Lambda_{m} \\
& \times\left(\frac{2 i-1}{M}-1\right) c_{n, m}, \\
& i=1,2,3, \ldots, M .
\end{aligned}
$$

The coefficients $c_{n, 0}$ and $c_{n, 2 l}$ can be calculated as:

$$
\begin{aligned}
& {\left[\begin{array}{cccc}
\frac{\sqrt{2}}{2} & \Lambda_{1}\left(\frac{1}{M}-1\right) & \ldots & \Lambda_{M-1}\left(\frac{1}{M}-1\right) \\
\frac{\sqrt{2}}{2} & \Lambda_{1}\left(\frac{3}{M}-1\right) & \ldots & \Lambda_{M-1}\left(\frac{3}{M}-1\right) \\
\vdots & \vdots & \ldots & \vdots \\
\frac{\sqrt{2}}{2} & \Lambda_{1}\left(\frac{2 M-1}{M}-1\right) & \ldots & \Lambda_{M-1}\left(\frac{2 M-1}{M}-1\right)
\end{array}\right]\left[\begin{array}{c}
c_{n, 0} \\
c_{n, 1} \\
\vdots \\
c_{n, M-1}
\end{array}\right]} \\
& =\sqrt{\frac{\pi}{2}} 2^{-\frac{k}{2}}\left[\begin{array}{c}
g\left(\frac{2(n-1) M+1}{2^{k} M}\right) \\
g\left(\frac{2(n-1) M+3}{2^{k} M}\right) \\
\vdots \\
g\left(\frac{2(n-1) M+2 M-1}{2^{k} M}\right)
\end{array}\right]
\end{aligned}
$$

For $\mathrm{M}=1$, the above equation can be evaluated as:

$$
c_{n, 0}=\sqrt{\pi} 2^{-\frac{k}{2}} g\left(\frac{2 n-1}{2^{k}}\right),
$$

Thus, Chebyshev wavelet based integration can be evaluated as follows:

$$
\int_{0}^{1} g(x) d x \simeq \frac{1}{2^{k-1}} \sum_{n=1}^{2^{k-1}} g\left(\frac{2 n-1}{2^{k}}\right)
$$


For $M=5$ :

$$
\begin{gathered}
\int_{0}^{1} g(x) d x=\frac{1}{9 \times 2^{k+6}} \sum_{n=1}^{2^{k+1}}\left[275 g\left(\frac{10 n-9}{5 \times 2^{k}}\right)\right. \\
+100 g\left(\frac{10 n-7}{5 \times 2^{k}}\right)+402 g\left(\frac{10 n-5}{5 \times 2^{k}}\right)+ \\
\left.100 g\left(\frac{10 n-3}{5 \times 2^{k}}\right)+275 g\left(\frac{10 n-1}{5 \times 2^{k}}\right)\right]
\end{gathered}
$$

For $M=6$,

$$
\begin{gathered}
\int_{0}^{1} g(x) d x=\frac{1}{5 \times 2^{k+7}} \sum_{n=1}^{2^{k+1}}\left[247 g\left(\frac{12 n-11}{3 \times 2^{k+1}}\right)+\right. \\
139 g\left(\frac{12 n-9}{3 \times 2^{k+1}}\right)+254 g\left(\frac{12 n-7}{3 \times 2^{k+1}}\right)+ \\
254 g\left(\frac{12 n-5}{3 \times 2^{k+1}}\right)+139 g\left(\frac{12 n-3}{3 \times 2^{k+1}}\right)+ \\
\left.247 g\left(\frac{12 n-1}{3 \times 2^{k+1}}\right)\right]
\end{gathered}
$$

For detailed discussion on Block Pulse function and Chebyshev wavelet based integration, the reader is referred to works $[23,27,28]$.

\subsection{Consistency}

A numerical method is called convergent if it approaches the exact solution as the discretization is refined. Consistency and stability guarantees convergence of the solution. The choice of monomial basis vector $\mathrm{p}(\mathrm{x})$ influences the consistency of the EFG method. MLS approximation reproduces all components that appear in $\mathrm{p}(\mathrm{x})$. EFG method is consistent of order $\mathrm{k}$ if all monomials up to order $k$ are included in $p(x)$ [29]. If $k=1$, this implies that linear complete, $k=2$, quadratic complete etc. For consistency test we consider MLS approximation which reproduces all components of polynomials.

$$
\begin{array}{lc}
\sum_{I=1}^{2^{n}} \Phi_{I}^{k}(x) u\left(x_{I}\right)=u^{k}(x) \quad \forall x \varepsilon \Omega \\
\text { If } u(x)=1, \text { then } & \sum_{I=1}^{2^{n}} \Phi_{I}(x)=1 \\
\text { If } u(x)=x \text {, then } & \sum_{I=1}^{2^{n}} \Phi_{I}(x) x_{l}=x
\end{array}
$$

\section{Numerical Examples}

The proposed improved EFG technique was tested on the following boundary value problems.

\section{Problem 3.1}

$$
\frac{d^{6} u}{d x^{6}}+e^{-x} u=-720+e^{-x}\left(x-x^{2}\right)^{3}, \quad 0 \leq x \leq 1
$$

Boundary conditions:

$$
u^{i}(0)=u^{i}(1)=0, \quad i=0,1,2
$$

Exact Solution [11]:

$$
u(x)=x^{3}(1-x)^{3}
$$

\section{Problem 3.2}

$$
\frac{d^{6} u}{d x^{6}}=x^{3}-3 x^{4}+3 x^{5}-x^{6}, 0 \leq x \leq 1
$$

With corresponding non-homogeneous boundary conditions:

$$
u^{i}(0)=u^{i}(1)=0, i=0,1,2
$$

Exact Solution:

$$
\begin{array}{r}
u(x)=\frac{1}{221760}\left(-\frac{1}{3} x^{12}+2 x^{11}-\frac{22}{5} x^{10}+\frac{11}{3} x^{9}\right) \\
+\frac{1}{10^{8}}\left(-2976 x^{5}+4058 x^{4}-1503 x^{3}\right)
\end{array}
$$

Test problems were solved for different number of nodes and types of basis with the help of improved EFG based on numerical integration using Chebyshev (CW) and Block pulse functions/wavelets (BPF). Maximum absolute errors in solution are tabulated and compared with the results of exact solution, which show better accuracy.

\subsection{Results and Discussion}

Maximum errors obtained using our technique based on Block Pulse Function (BPF) and Chebyshev wavelet for our test problems are shown in the following Tables and figures. Comparison of Maximum absolute errors with exact solution, demonstrates the accuracy of our method as shown in the Tables 1, 2 and figures 3-14.

\subsection{Effect of Integration points}

It has been observed, that the accuracy of meshless computational technique depends upon the size of influence of domain, penalty factor and as well as Integration points. In this paper we have observed that, 


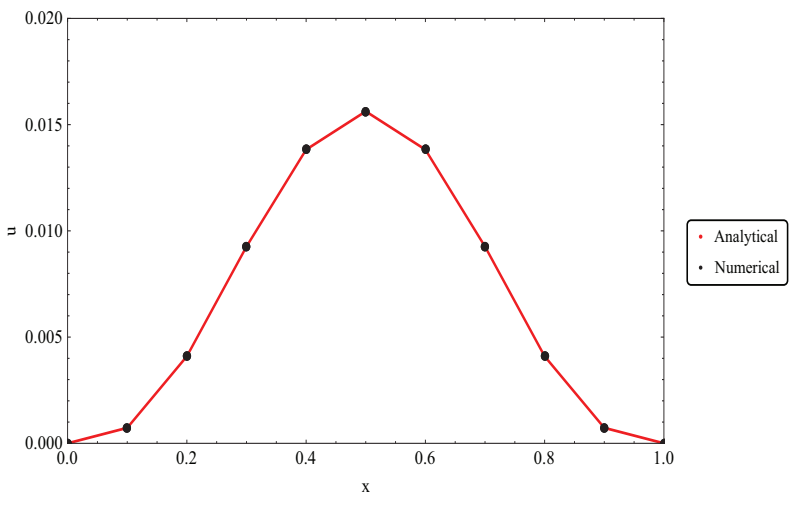

Fig. 3: Numerical and Analytical Solution Plot of u (6 nodes), Problem 3.1

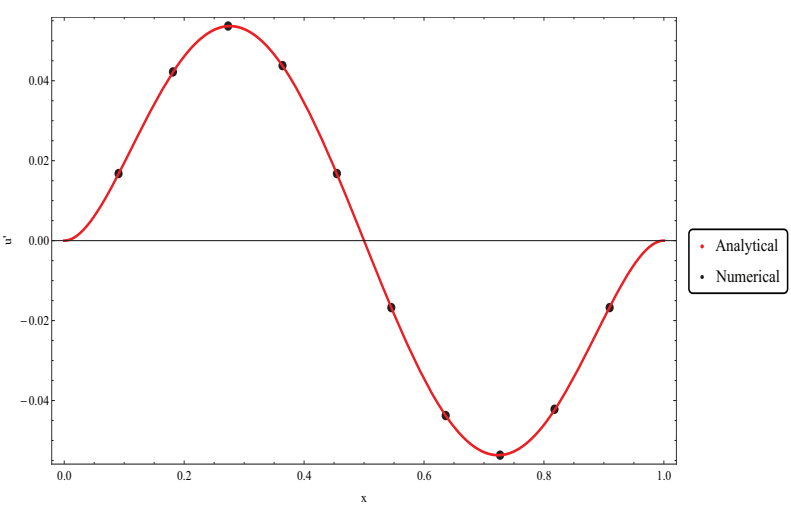

Fig. 4: Numerical and Analytical Solution Plot of $\frac{d u}{d x}(6$ nodes $)$, Problem 3.1

Table 1: Results for Problem 3.1

\begin{tabular}{|l|l|l|}
\hline $\begin{array}{l}\text { Numerical } \\
\text { Integration } \\
\text { Technique } \\
\text { for EFG }\end{array}$ & $\begin{array}{l}\text { Number } \\
\text { of } \\
\text { nodes }\end{array}$ & $\begin{array}{l}\text { Max.Absolute } \\
\text { Error }\end{array}$ \\
\hline BPF(22) & 6 & $1.51392 \mathrm{E}-7$ \\
CW(23) & 6 & $3.499 \mathrm{E}-13$ \\
CW(24) & 6 & $4.673 \mathrm{E}-14$ \\
\hline
\end{tabular}

Table 2: Results for Problem 3.2

\begin{tabular}{|l|l|l|}
\hline $\begin{array}{l}\text { Numerical } \\
\text { Integration } \\
\text { Technique } \\
\text { for EFG }\end{array}$ & $\begin{array}{l}\text { Number } \\
\text { of } \\
\text { nodes }\end{array}$ & $\begin{array}{l}\text { Max.Absolute } \\
\text { Error }\end{array}$ \\
\hline BPF(22) & 6 & $1.246 \mathrm{E}-9$ \\
CW(23) & 6 & $1.246 \mathrm{E}-9$ \\
CW(24) & 6 & $1.246 \mathrm{E}-9$ \\
\hline
\end{tabular}

the accuracy of numerical method increases as we

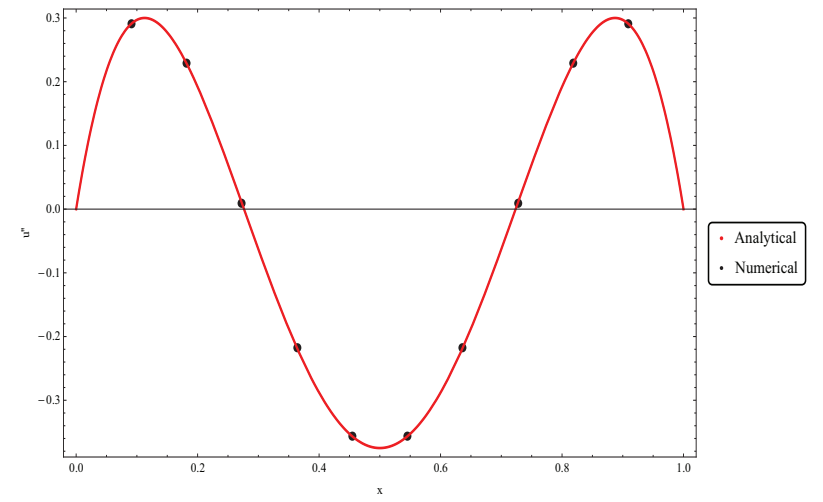

Fig. 5: Numerical and Analytical Solution Plot of $\frac{d^{2} u}{d x^{2}}$ (6 nodes), Problem 3.1

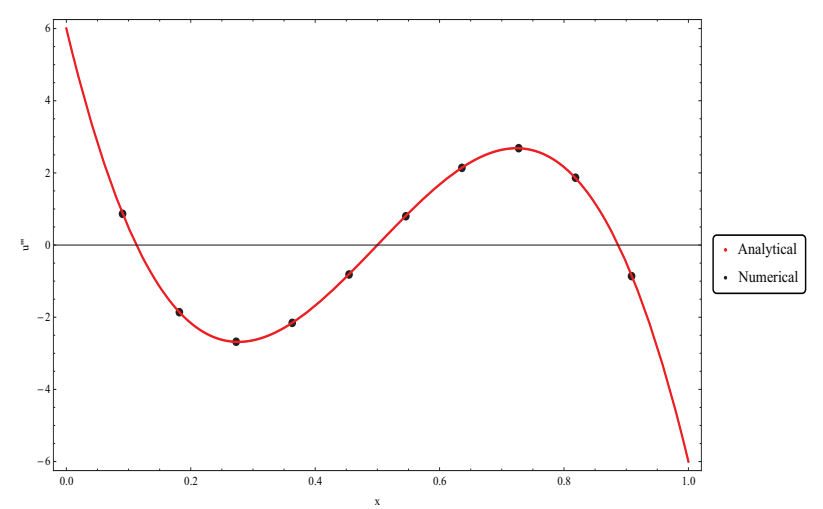

Fig. 6: Numerical and Analytical Solution Plot of $\frac{d^{3} u}{d x^{3}}$ (6 nodes), Problem 3.1

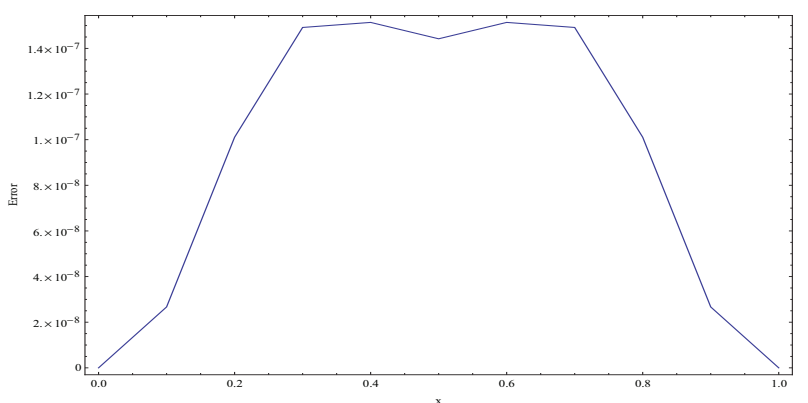

Fig. 7: Max. Absolute Error of Numerical (22) and Analytical Solution Plot (6 nodes), Problem 3.1

increase the number of integration points, however if the number of gauss points exceeds some limiting value, it may have some adverse effects on the accuracy. It has been determined that arrangement of background cells and ratio of total number of Gauss points to the total number of nodes, plays very important roles in accuracy. To avoid all these complications and to improve the 


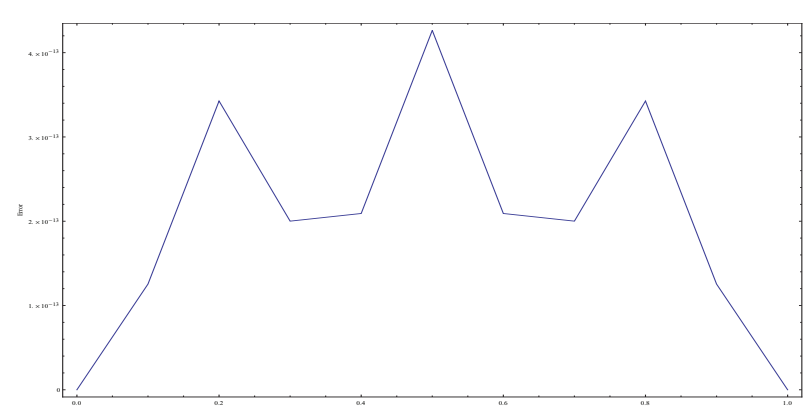

Fig. 8: Max. Absolute Error of Numerical (23) and Analytical Solution Plot (6 nodes), Problem 3.1

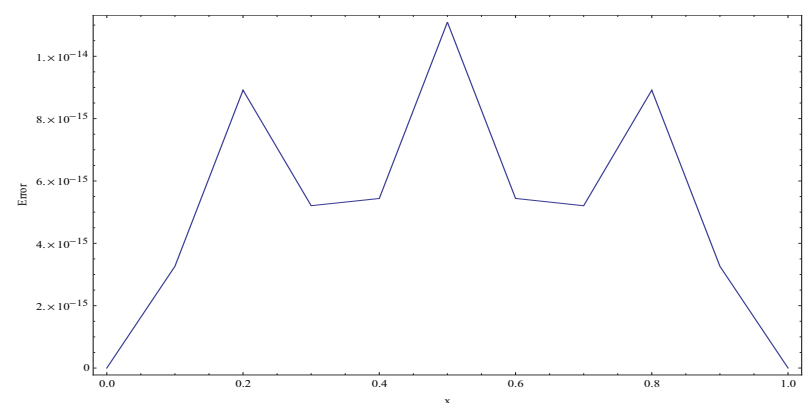

Fig. 9: Max. Absolute Error of Numerical (24) and Analytical Solution Plot (6 nodes), Problem 3.1

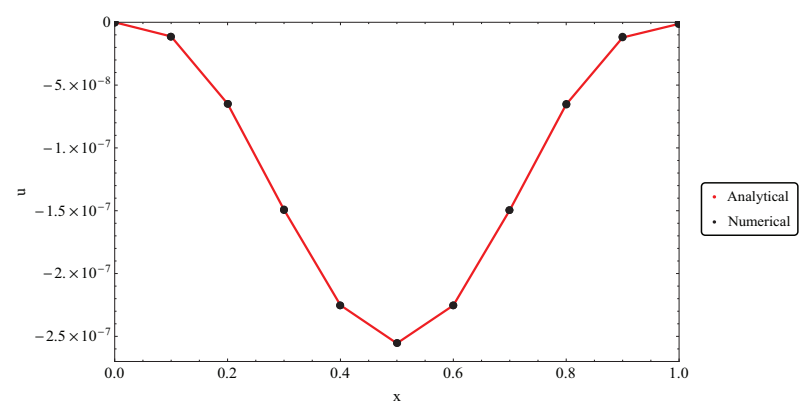

Fig. 10: Numerical and Analytical Solution Plot of u (6 nodes), Problem 3.2

accuracy of the meshless method, we have purpose new numerical integration technique as discussed.

\section{Conclusion}

In this study, novel numerical technique Element free Galerkin (EFG) technique using Block-Pulse function and Chebchieve Wavlet based Integration has been presented. The results obtained by the suggested method for problem 3.1 and 3.2, as mentioned above exhibit its ability to provide improved solutions as compared to results in

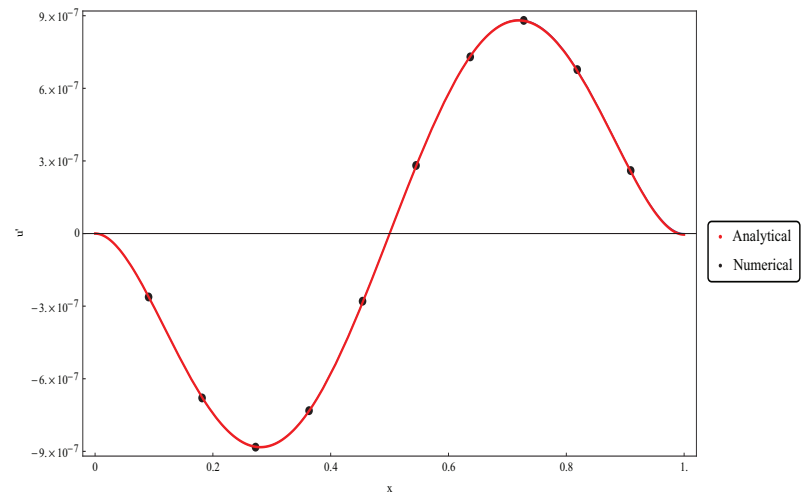

Fig. 11: Numerical and Analytical Solution Plot of $\frac{d u}{d x}(6$ nodes), Problem 3.2

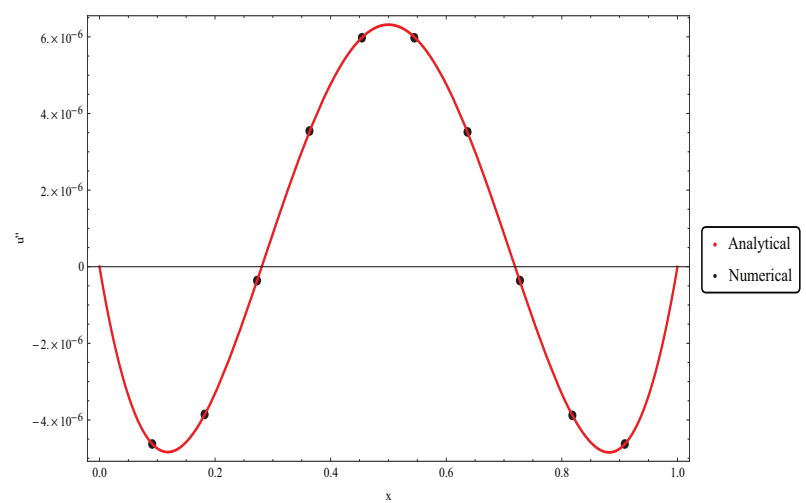

Fig. 12: Numerical and Analytical Solution Plot of $\frac{d^{2} u}{d x^{2}}$ (6 nodes), Problem 3.2

Table 3: Results for Problem 3.2

\begin{tabular}{|l|l|l|l|}
\hline $\mathrm{x}$ & $\begin{array}{l}\text { Exact } \\
\text { Solution }\end{array}$ & $\begin{array}{l}\text { Sinc- } \\
\text { Galerkin } \\
{[11]}\end{array}$ & $\begin{array}{l}\text { Max. } \\
\text { Absolute } \\
\text { Relative } \\
\text { Error, } \\
1.0 \mathrm{E}-3\end{array}$ \\
\hline 0.0 & 0.0 & 0.0 & - \\
0.1675 & 0.002711 & 0.002710 & 0.45 \\
0.2764 & 0.008000 & 0.008997 & 0.32 \\
0.3449 & 0.011534 & 0.011531 & 0.28 \\
0.4205 & 0.014469 & 0.014465 & 0.26 \\
0.5 & 0.015625 & 0.015620 & 0.25 \\
0.6550 & 0.011539 & 0.011536 & 0.28 \\
0.7828 & 0.004915 & 0.004913 & 0.37 \\
0.8324 & 0.002715 & 0.002714 & 0.45 \\
0.9041 & 0.000651 & 0.000651 & 0.73 \\
1.0 & 0.0 & 0.0 & - \\
\hline
\end{tabular}

[11]. The improved EFG technique showed fast convergence and provided better results at reduced number of nodes. It has been observed that EFG based on 


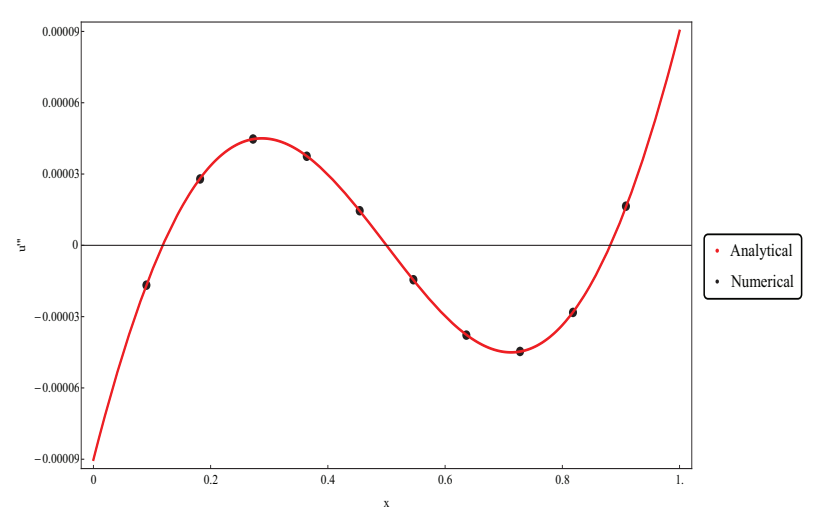

Fig. 13: Numerical and Analytical Solution Plot of $\frac{d^{3} u}{d x^{3}}$ (6 nodes), Problem 3.2

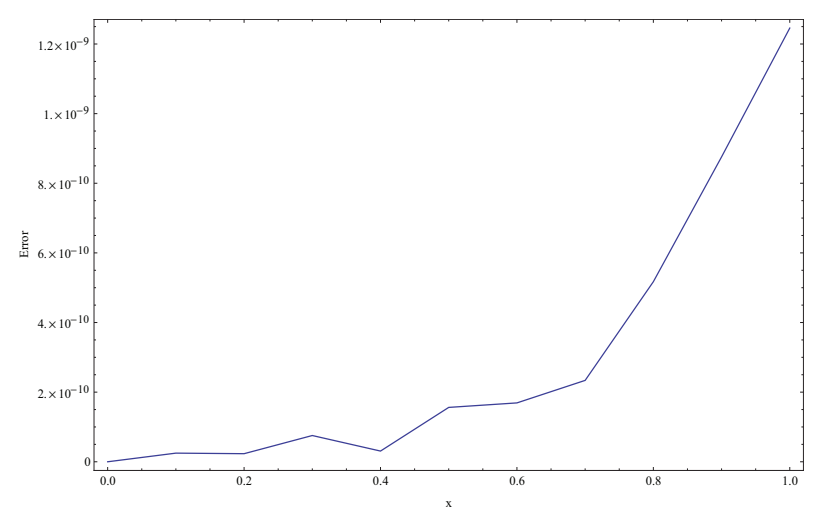

Fig. 14: Error Plot (6 nodes), Problem 3.2

Numerical integration techniques is faster than existing techniques, and has the capability of handling of improper, highly oscillatory functions.

\section{Acknowledgement}

The authors are grateful to the anonymous referee for a careful checking of the details and for helpful comments that improved this paper.

\section{References}

[1] P. Baldwin, A localized instability in a Bernard layer, Applicable Analysis, 24, 117-156 (1987).

[2] P. Baldwin, Asymptotic estimates of the eigenvalues of a sixth-order boundary-value problem obtained by using global-phase integral methods, Phil. Trans. R.Soc. Land A, 322, 281-305 (1987).

[3] G. A. Glatzmaier, Numerical simulations of stellar convective dynamics III: at the base of the convection zone, Geophysics and Astrophysics Fluid Dynamics, 31, 137-150 (1985).
[4] J. Toomre, J. R. Zahn, J. Latour and E. A. Spiegel, Stellar convection theory II: singlemode study of the second convection zone in A-type stars, Astrophys. J., 207, 545-563 (1976).

[5] S. Chandrasekhar, Hydrodynamic and Hydro magnetic Stability, Oxford: Clarendon Press, 1961, (Reprinted: Dover Books, New York, 1981).

[6] E. H. Twizell, A second order convergent method for sixthorder boundary-value problems, in Numerical Mathematics, Singapore 1988 (Agarwal, R. P., Chow. Y. M. and Wilson, S. J., eds). Birkhhauser Verlag, Basel, 495-506 (1988).

[7] E. H. Twizell and Boutayeb, Numerical methods for the solution of special and general sixth-order boundary-value problems, with applications to Benard layer eigenvalue problems. Proc. R. Soc. Land. A., 431, 433-450 (1990).

[8] R. P. Agarwal, Boundary-Value Problems for Higher-Order Differential Equations, World Scientific, Singapore, (1986).

[9] G. Akram and S. S. Siddiqi, Solution of linear sixthorder boundary-value problems using non-polynomial spline technique, Appl. Math. Computation, 1, 708-720 (2006).

[10] M. M. Chawala and C.P Katti, Finite difference methods for two-point, boundary-value problems involving higher-order differential equations, BIT, 19, 27-33 (1979).

[11] M. E. Gamel, J. R. Cannon, J. Latour A.I Zayed, SincGalerkin Method for solving linear sixth-order boundaryvalue problems, Math. Computer, 73, 1325-1343 (2003).

[12] Siraj-ul-Islam, Ikram A. Tirmizi, Fazal-i-Haq, M. Azam Khan, Non-Polynomial Splines Approach to the Solution of Sixth-Order Boundary-Value Problems, 195, 270-284 (2008)

[13] A. M. Wazwaz, The numerical solution of sixth-order boundary-value problems by the modified decomposition method, Appl. Math. Comput., 118, 311-325 (2001).

[14] A. M. Wazwaz, The modified Adomain decomposition method for solving linear and nonlinear boundary value problems of 10th-order and 12th-order. Int. J. nonlin.Sci. Numer. Simu., 1, 17-24 (2000).

[15] J. H. He variational approach to the sixth-order boundary value problems. Appl. Math. Comput., 143, 537-538 (2003).

[16] J. H. He. Semi-inverse method of establishing generalized variational principles for fluid mechanics with emphasis on turbomachinery aerodynamics, In.J. Turbo \& Jet-Engines, 14, 23-28 (1997).

[17] S. S. Siddiqi and E. H. Twizell, Spline solutions of linear sixth-order boundary-value problems, Intern. J. Computer Math., 45, 295-304 (1996).

[18] Atluri, S. N., Zhu, T. A new meshless local petrov-galerkin (MLPG) approach in computational mechanics. Comput. Mech., 22, 117-127 (1998).

[19] Satya N. Atluri. Methods of computer modeling in engineering and the sciences, Tech science press, I, (2005).

[20] Atluri, S. N., Zhu, T. New concepts in meshless methods. Int. J. Nu. Meth. Eng., 47, 537-556 (2000).

[21] G. R. Liu. Mesh Free Methods: Moving beyond the Finite Element Method CRC Press LLC, (2003).

[22] Belytschko T, Lu YY, Gu L, Element-Free Galerkin methods Int. J. Numer. Methods Engrg., 37, 229-256 (1994).

[23] Majid Rostami, Elham Hashemizadeh, Mohammd Heidari. A comparative study of numerical integration based on block-pulse and sinc functions and Chebyshev wavelet. Mathematical Sciences, 8, (2012) 
[24] Rabbani, M, Nouri, K. Solution of integral equations by using block-pulse functions. Mathematical Sciences Quarterly Journal (MSQJ), 4, 39-48 (2010).

[25] K. Surla,. D. Herceg and L. Cvetkovic, Solving singularlyperturbed boundary-value problems by splines in tension, J. Computational and applied mathematics, 24, 355-363 (1988).

[26] K. Surla and M. Stojanovic, A finally of exponential spline difference scheme, review of research, faculty of science, mathematics series, University of Novi Sad, 19, 12-23 (1991).

[27] Prem K. Kythe. Michael R. Schaferkotter. Hand Book of Computational Methods for Integration, (2005).

[28] Stenger F., Numerical methods based on Sinc and Analytic functions. Springer Verlag,New York, (1993).

[29] Krysl P. and T. Belytschko, Analysis of Thin Shells by the Element-Free Galerkin Method, Int. J. Solids and Structures, 33, 3057-3080 (1996).

[30] A. Boutayeb and E. H. Twizell, Numerical methods for the solution of special sixth-order boundary-value problems, Intern. J. Computer Math., 45, 207-223 (1992).

[31] Mazzia A, Ferronato M, Pini G, Gambolati G. A comparison of numerical integration rules for the meshless local PetrovGalerkin method. Numer Algorithms, 61, 61-74 (2007).

[32] G. R. Liu, G. Y. Zhang, Y. Y. wang ,Z. H zhong, G. Y. $\mathrm{Li}, \mathrm{X}$ Han A nodal integration technique for meshfree radial point interpolation method international journal of solids and structures, 44, 3840-3860 (2007).

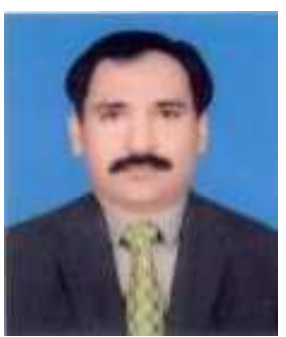
Muhammad
Azam
is involved in the research
on numerical techniques
for over a decade. $\mathrm{He}$
has done his masters
in Engineering Sciences from
GIKI, Pakistan. His research
interests are numerical analysis, computational techniques, structural analysis and fluid mechanics. His doctoral research area is related to meshfree techniques. He has published his research on numerical techniques in several international journals of repute.

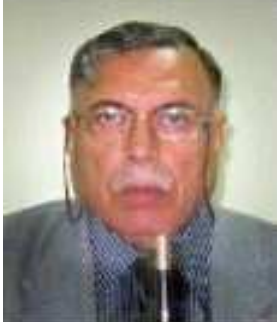

Khalid Parvez is Professor in Department of Aeronautics and Astronautics, Institute of Space Technology, Islamabad, Pakistan. Previously he was Professor in Research Center for Modeling and Simulation (RCMS) and College of Aeronautical Engineering (CAE), National University of Sciences and Technology, Pakistan. His research interests are modeling and simulation, turbomachinery and combustion. He has published in many journals of international repute.

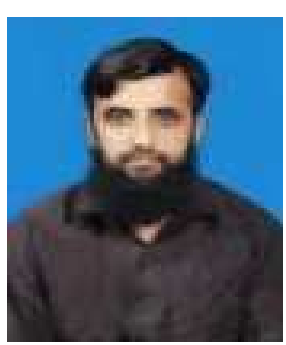

Muhammad Omair is involved in the field of Finite Element Analysis for a period of over 8 years. He has done his masters in Computational Science and Engineering from Research Center for Modeling and Simulation (RCMS), National University of Sciences and Technology, Pakistan. His research interests include computational mechanics of fluids and structures and meshfree techniques. He has published and presented in many international journals and conferences. 